\title{
Laser Peening of Metals - Enabling Laser Technology
}

\author{
C. Brent Dane \\ L. A. Hackel \\ J. Daly \\ J. Harrisson
}

This paper was prepared for submittal to the Materials Research Society 1997 Fall Meeting

Boston, MA

December 1-5, 1997

November 13, 1997

This is a preprint of a paper intended for publication in a journal or proceedings. Since changes may be made before publication, this preprint is made available with the understanding that it will not be cited or reproduced without the permission of the author. 


\section{DISCLAIMER}

This document was prepared as an account of work sponsored by an agency of the United States Government. Neither the United States Government nor the University of California nor any of their employees, makes any warranty, express or implied, or assumes any legal liability or responsibility for the accuracy, completeness, or usefulness of any information, apparatus, product, or process

disclosed, or represents that its use would not infringe privately owned rights. Reference herein to any specific commercial product, process, or service by trade name, trademark, manufacturer, or otherwise, does not necessarily constitute or imply its endorsement, recommendation, or favoring by the United States Government or the University of California. The views and opinions of authors expressed herein do not necessarily state or reflect those of the United States Government or the University of California, and shall not be used for advertising or product endorsement purposes. 


\title{
Laser Peening of Metals - Enabling Laser Technology
}

\author{
C. Brent Dane and Lloyd A. Hackel \\ Lawrence Livermore National Laboratory, Livermore CA 94550 \\ James Daly and James Harrisson \\ Metal Improvement Corporation, Paramus NJ
}

\section{Introduction}

Laser peening, a surface treatment, for metals, employs laser induced shocks to create deep and intense residual stresses in critical components. In many applications this technology is proving to be superior to conventional treatments such as shot peening. The laser peening process has generated sufficiently impressive results to move it from a laboratory demonstration phase into a significant industrial process. However until now this evolution has been slowed because a laser system meeting the average power requirements for a high throughput process has been lacking.

A laser system appropriate for peening at an industrial level requires an average power in the multi-hundred watt to killowatt range and an energy of around $100 \mathrm{~J} /$ pulse and pulse duration of 10 s of nanoseconds. Pulsed lasers, with output energies exceeding $10 \mathrm{~J}$, have historically been limited to low repetition rates and consequent low average power output. The large fusion lasers such as Livermore's Nova and the University of Rochester's Omega laser can produce single pulse energies at 1 micron wavelength in the $100 \mathrm{~kJ}$ range but are limited to firing about once every two hours for an equivalent average power of only 10 s of watts. Commercially available lasers, with outputs of 10 to $100 \mathrm{~J}$, if available at all, 
are limited to repetition rates around $0.25 \mathrm{~Hz}$, an average power of $25 \mathrm{~W}$. In this paper we report on a highly developed laser technology employing Nd:glass slabs and a master oscillator/power amplifier with wavefront correction called phase conjugation which for the first time pushes the average power output into the 500 $\mathrm{W}$ range and meets the requirements for industrial laser peening.

\section{Laser Shock Peening}

With the invention of the laser, it was rapidly recognized that the intense shocks required for peening could be achieved by means of tamped plasmas which were generated at metal surfaces by means of high energy density $\left(200 \mathrm{~J} / \mathrm{cm}^{2}\right)$, lasers with pulselengths in the tens of nanoseconds range. Initial studies on laser shock processing of materials were done at the Battelle Institute (Columbus, $\mathrm{OH}$ ) from about 1968 to $1981^{1,2}$. Excellent recent work has also been reported in France ${ }^{3}$. Figure 1 shows a typical setup for laser peening. Laser intensities of $100 \mathrm{~J} / \mathrm{cm}^{2}$ to 300 $\mathrm{J} / \mathrm{cm}^{2}$ in a pulse duration of about $30 \mathrm{~ns}$ can generate shock pressures of $10^{4}$ to $10^{5}$ atmospheres when absorbed on a metal surface ( $a$ thin layer of black paint on the surface provides an excellent absorber and inertially confined with a surface layer (tamp) such as water. These shocks have been shown to impart compressive stresses, deeper than those achievable with standard shot 


\section{Laser input at $\sim 200 \mathrm{~J} / \mathrm{cm}^{2}$}

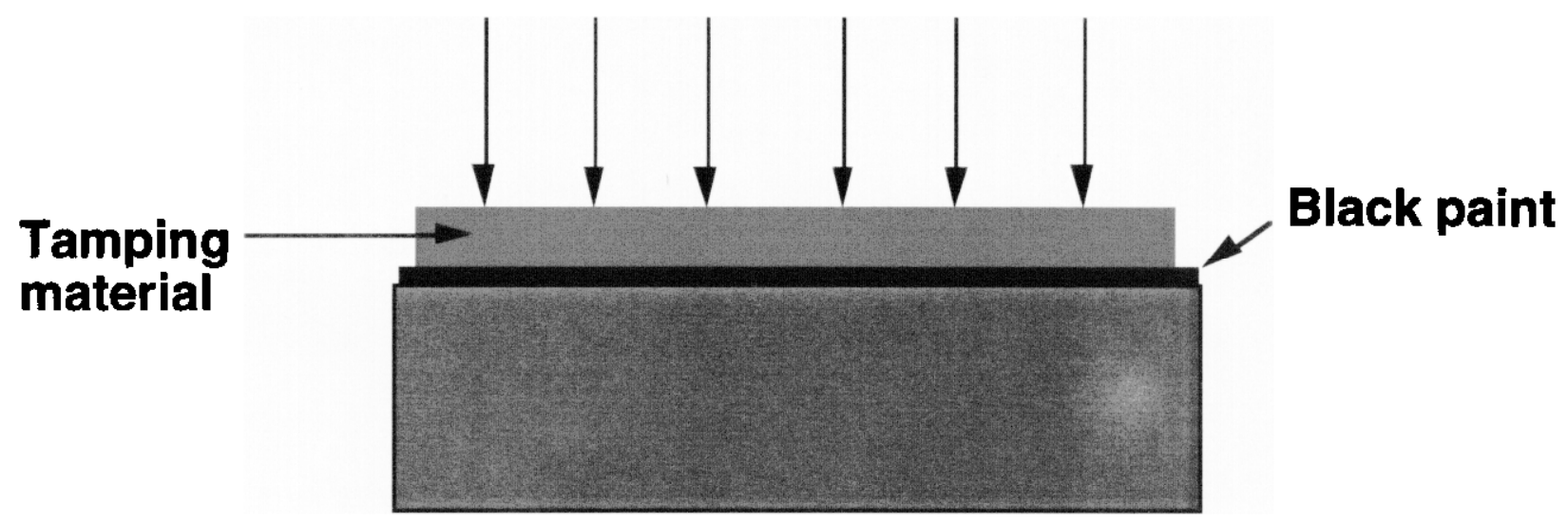

Figure 1. Typical setup for laser peening including a input laser beam of $200 \mathrm{~J} / \mathrm{cm} 2$ and $30 \mathrm{~ns}$ pulselength. The metal layer is covered with a layer of paint to provide light absorption and is covered with a thin water tamping layer to contain the shock.

peening. Special techniques in controlling the pulse temporal and spatial shape are used to prevent the high intensity laser from breaking down the water column or generating stimulated processes which reflect the laser energy before reaching the paint surface. With appropriate care given to the setup, impressive results can be achieved from laser peening.

Figure 2 shows the residual stress induced in Inconnel (Ti-6Al-4V) by laser peening and contrasts it with typical results achieved by shot peening. Clearly the laser generated shock can be tailored to penetrate deeper into the material and create a significantly greater stress volume. Induced residual stress prevents parts from developing cracks due to stress corrosion. Additionally other types of corrision will require longer periods of time to penetrate the compression layer induced by Lasershot Peening. Deep residual stress is important in components such as turbine blades because it prevents debris damage from penetrating beneath the compressive 
layer. Foreign object debris (FOD) picked up in operation can often generate damage sites which penetrate a thinner compressive layer and hence become an initiation point for fatigue cracks.



Figure 2. Residual stress induced by laser peening can be made deeper and with significantly greater stressed volume than conventional shot peening. 
Another important element in obtaining deep residual stresses is the use of successive shocks to drive the stress deeper and deeper while not exceeding materials limits at the surface. Figure 3 shows results of successive applications to a titanium surface (Ti-6Al-4V) of laser pulses at $200 \mathrm{~J} / \mathrm{cm}^{2}$ and pulse duration of $30 \mathrm{~ns}$. As can be seen, the application of a first and then a second shock drives stress deeper and into the material.

Figure 3. Comparison of residual stress resulting from a single and then a second laser peeing pulse

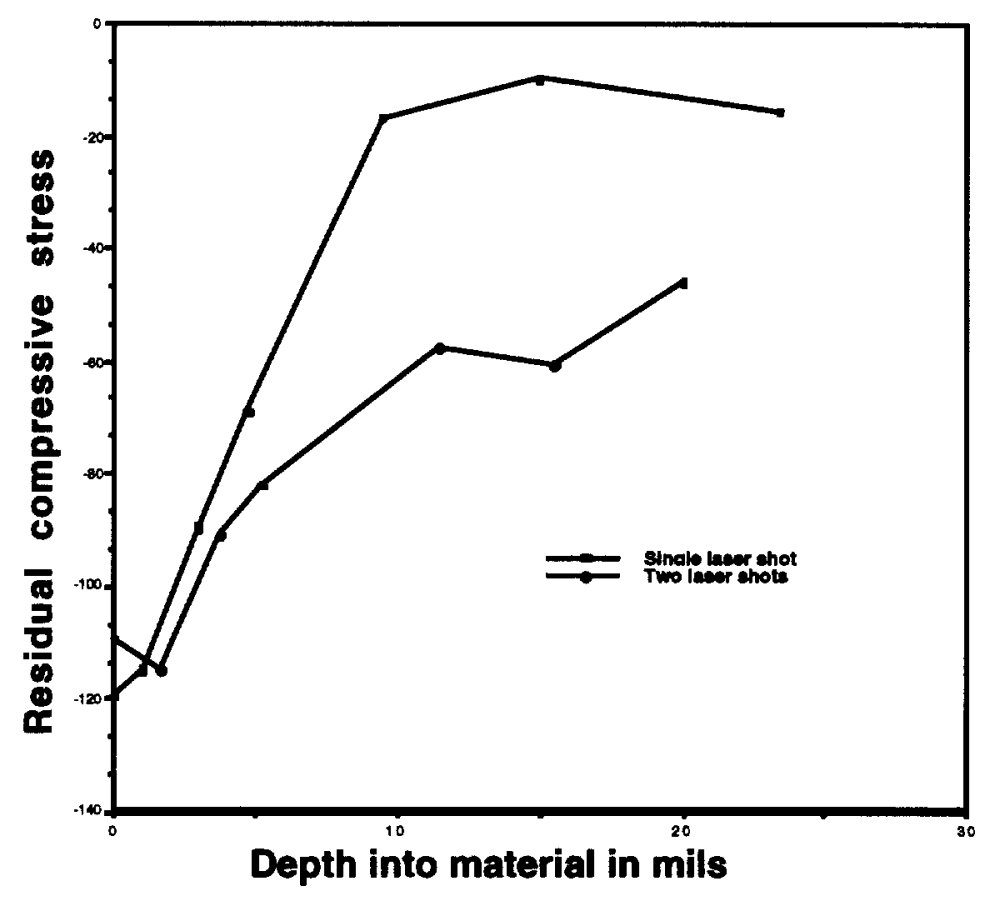

Figure 3. A first and then second lasershot pulse can generate successively deeper residual stress. 
In testing on operational components, such as jet engine fan blades, researchers have shown this treatment to be superior for strengthening new and previously damaged fan blades from fatigue and corrosion failure ${ }^{4}$. However the laser technology for doing these types of tests has been limited to producing pulses less than once per second thus peening areas of about 1 square centimeter per second. This rate is acceptable for laboratory demonstrations but clearly not meaningful for production. Additionally since the cost of any high energy (100 J) laser is dominated by the hardware required to achieve the single pulse energy, it is imperative to have high repetition rate capability $(\sim 10 \mathrm{~Hz})$ in order to keep the production cost per laser shot low.

\section{High power laser technology at LLNL}

The Laser Programs Directorate at Lawrence Livermore National Laboratory (LLNL) has been a world leader in developing high energy Nd:glass lasers for fusion applications for the past 25 years. The Nova laser, producing over $120 \mathrm{~kJ}$ per pulse, routinely fires 8 to 10 shots per day for dedicated fusion and nuclear effects studies. More recently, the Livermore Laboratory has been directed by the Department of Energy to proceed with building a newer facility, the National Ignition Facility (NIF) which will produce over $2 \mathrm{MJ}$ per pulse of energy in one or several shots per day and is intended to produce more fusion energy release than laser energy input.

Generating high energy from a solid state glass laser is not an issue. However generating high average power at high energy has been. Over the past decade, LLNL has been developing higher average power systems with energies of $25 \mathrm{~J}$ to 100 $\mathrm{J} /$ pulse. The laser technology has repetition rates of up to $10 \mathrm{~Hz}$ and average powers near $1 \mathrm{~kW}$. The technology has been supported by the Advanced Research Projects Agency (ARPA) of the Department of Defense and more recently by the US Navy and US Air Force. The ARPA funding was focused on converting the infrared light 
to high average power $x$-rays $(10 \AA)$. This short wavelength has interest as a light source for printing advanced generation integrated circuits. The Navy and Air Force funding was directed toward obtaining a light source for long range and highly coherent illumination of missiles and space objects. One of our LLNL lasers is currently in service at a Navy facility at the Kennedy Space Center, Cape Canaveral Florida and a second more power unit is being delivered to the Air Force Phillips Laboratory, Albuquerque New Mexico.' This technology has allowed us to develop a glass laser system with energy of $100 \mathrm{~J} /$ pulse, adjustable pulse length from $10 \mathrm{~ns}$ to 1 $\mu \mathrm{s}$, near diffraction limited beam quality and average power up to $600 \mathrm{~W}$. This laser technology is ideal for the laser peening application and by a factor of 20 to 50 exceeds the average power achieveable by any commercially available laser technology.

\section{The High Average Power Nd:glass Slab Laser System}

A system suitable for laser peening must output an energy in the range of $25 \mathrm{~J}$ to $100 \mathrm{~J}$ per pulse. The throughput of a peening system will then highly depend on the average pulse repetition rate that the laser can achieve. A laser system based on $\mathrm{Nd}$ doped glass gain media is the only identified technology that can realistically achieve this type of energy output with acceptable pulselength. Such a system is typically based on an oscillator and one or more rod amplifiers which are optically pumped by flashlamps. As an unavoidable consequence of providing the optical gain, the flashlamps deposit heat into the glass. This heat must be removed at a rate commensurate with the rate of deposition, that is, the pulse rate of the laser. Thus the glass must be cooled, typically by flowing water. As the glass is simultaneously heated and cooled a thermal gradient develops from the center to edge of the glass. This gradient stresses the glass, inducing wavefront deformation and very significantly depolarization of the beam. Thus the thermal loading of the laser gain 
media is a major limitation to the available average power that can be extracted from the laser. As the repetition rate of the laser is increased, the thermal loading correspondingly increases and degenerates the laser performance often depolarizing and aberrating the laser beam to the point where the laser optics damage. In the limit, the loading will fracture the glass. The LLNL laser system alleviates this thermal problem in three ways; the slab gain medium is pumped in a highly uniform manner minimizing depolarization and distortion, the laser beam is propagated through the slab in a zig-zag manner to average out much of the wavefront distortion and SBS phase conjugation highly corrects residual wavefront distortions.

\section{The LLNL Nd:glass slab laser system}

The LLNL high average power Nd:glass laser technology is comprised of a single master oscillator and one or more power amplifiers. The amplifier gain media is neodymium $(\mathrm{Nd})$ doped phosphate glass APG1 supplied by Schott Glass Technologies Inc. or HAP4 supplied by Hoya Corporation. The glass is configured in a slab shape to allow one thin dimension for rapid heat removal. Typical slab dimensions are $1 \mathrm{~cm} \times 14 \mathrm{~cm}$ by $40 \mathrm{~cm}$. Typical Nd doping level is $3 \times 10^{20} \mathrm{~cm}^{-3}$ or $2.7 \%$ by weight.. Figure 4 shows a cross-sectional view of the amplifier.

Unlike a more traditional amplifier where the beam is propagated in a straight line through the amplifier, our design employs a zig-zag path path, reflecting the beam internally off the slab faces, to propagate it through the gain medium. The large one-dimensional temperature gradients through the slab 
thickness are effectively averaged by the zig-zag optical path as shown in Figure 4 . The slab is positioned in the center of the assembly and has a water cooling channel along both sides formed by the slab face and a reflector window. Two flashlamps on each side pump the slab through the cooling channels. A diffuse reflector surrounds the flashlamps and by appropriate shaping provides uniform optical pumping of the slab. The reflector material is made of a Teflon-like substance called Spectralon machined to a specific shape to tailor the pumping irradiance on the slab surfaces. Designing one thin dimension for the slab creates one short path for high heat conduction from the slab center to the cooling water. The resulting high heat transfer efficiently removes the heat buildup in the slab and directly increases the repetition rate capability of the laser. Very uniform optical pumping from the reflector assembly results in uniform energy distribution from top to bottom in the slab. At high repetition rates the main thermal gradient that develops in the slab is from center to edge. However, the laser light is propagated through slab so that the beam propagates in a side-to-side zig-zag manner. This zig-zaging averages the side-to-side thermally induced pathlength differences providing a high quality horizontal wavefront even though there is a significant gradient in this direction. 
Slab amplifier operating in the AlT laser system

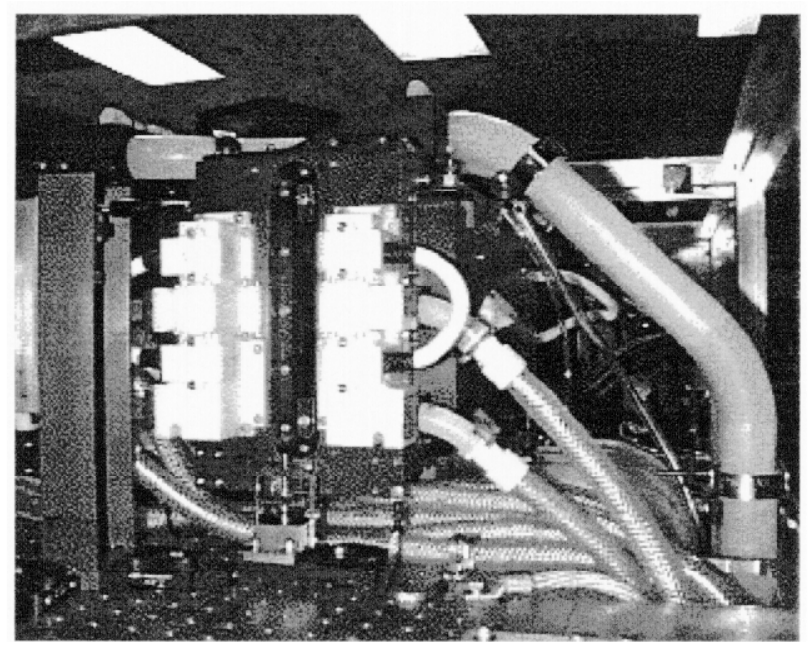

\section{Schematic of the amplifier design}

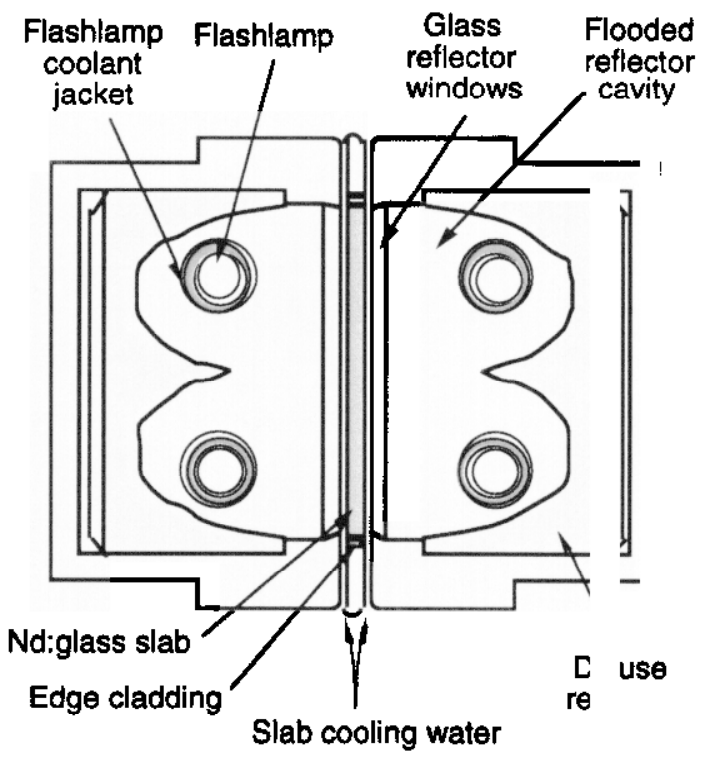

Figure 4. Photograph and cross-sectional view of the Nd:glass laser amplifier. Flashlamp light, tailored for highly uniform illumination by the diffuse reflectors, provides the excitation to the slab. The thin dimension of the slab allows for efficient heat extraction into the water flow. The laser light zig-zags through the slab averaging wavefront distortions.

The typical optical layout for the laser system is shown in figure 5. The output of the oscillator transmits through a Faraday isolator and then in $\mathrm{P}$ polarization transmits through a polarizing beam splitter. The beam next passes through an input/output Faraday rotator which acts as a passive cavity switch and then still in $\mathrm{P}$ polarization transits through the ring polarizer. It next transits a relay telescope, double passes the amplifier and then propagates back through the telescope and trough a 90 degree rotator. The now $S$ polarized beam reflects off the polarizing beam splitters, through the relay telescope and for a second time double passes the amplifier. Passing again through the 90 degree rotator and converting back to $\mathrm{P}$ polarization, the now propagates to SBS phase conjugator. The phase 
conjugator acts as a mirror, reflecting the beam back through the amplifier ring retracing its path back to the input/output Faraday rotator. In the output direction the rotator flips the beam to $S$ polarization, allowing it to exit the laser. In these final four passes, the beam amplifies to the desired output energy and very importantly, all phase errors accumulated in the first four amplifier passes are negated in the final four amplifier passes (by summation of phases which are basically identical but revrsed in sign) to generate a high power beam with nearly diffraction limited beam quality. Depending on the specific design, this energy is in the range of $25 \mathrm{~J}$ to $100 \mathrm{~J}$ or more.



Figure 5. Typical layout of the high energy Nd:glass laser system with repetition rate of 6 to $12 \mathrm{~Hz}$. The high quality master oscillator output is amplifier by means of 8 
passes through the slab amplifier. The SBS phase conjugator provides necessary wavefront correction so that the output is near diffraction limited beam quality.

The most important advantage of operating the amplifier system with an SBS mirror lies, of course, is its wavefront reversal properties. Correcting for thermal aberrations allows average power output up to the mechanical limit of the gain media. The SBS cell effectively conjugates the phase of the input beam producing a wavefront reversed output. As this output beam retraces the path of the input beam through four additional gain passes, the phase errors cancel and the high quality wavefront of the injected beam is recovered in the high energy output. There are additional significant advantages to the operation of the amplifier system with the SBS phase conjugator. Eight gain passes through the zig-zag slab amplifier can be achieved using passive polarization switching in the regenerative amplifier ring. The fact that the SBS cell provides interstage gain isolation makes this possible since, if it were replaced with a mirror, the small signal gain through eight consecutive gain passes would result in possible parasitic oscillation from the small reflective lossses of AR coated optical surfaces in the ring or in the output beam.

The SBS phase conjugator very effectively conjugates the first order aberration of tilt. This greatly reduces the sensitivity of the system performance to small changes of optical alignment in the ring. No change in output power or pointing direction during operation are observed for large mirror misalignments in the ring, limited only to those angular excursions that result in vignetting of the beams at the edges of the amplifier slab. 
Finally and specifically for the laser shock peening application, the SBS phase conjugation plays a significant role in preparing a fast rising edge laser pulse to avoid optical breakdown in the tamping material reducing the intensity of the shock. Because the SBS is a non-linear process with a definite threshold, the phase conjugator does not respond to the initial low intensity buildup typically associated with a laser pulse sharpening the leading edge of the laser pulse. The beam returned by the conjugator has its leading edge "clipped" and thus the returned pulseshape has a sharp, sub-nanosecond rising edge. The fast rising pulse is critically important for laser peening because it reduces the possibility of breakdown or other non-linear processing occurring in the tamping material and allows the full pulse energy to reach the paint area on the metal and contribute to building the high intensity shock.

Although not needed for shock peening, the laser's high output beam quality enables efficient conversion of the infrared output light into green light. Conversion efficiencies for this laser technology range from $65 \%$ at $1 \mu$ s duration pulses to over $80 \%$ for $10 \mathrm{~ns}$ pulses.

The output energy of the laser is limited by the physical size of the gain media, the saturation fluence of the material and the damage fluence that can be accommodated. Increasing the height of the slab becomes impractical due to the cost of large optics and increasing the length effects the gain and amplified spontaneous emission limits. Increasing the thickness directly decreases the average power capability. However using the newly demonstrated technique of phase locking multiple apertures we can scale the laser output into exciting new levels of energy and average power. In this technique a single laser oscillator feeds multiple laser amplifiers and the beams are recombined into a single phase conjugator which 
effectively locks the separate channels into a single coherent laser beam. The far field beam quality is near the physical diffraction limit, the laser energy is that of the combined multiple apertures and the repetition rate is the higher one associated with a single laser slab. Figure 6 shows a highly engineered $100 \mathrm{~J} /$ pulse glass slab laser system packaged and ready for deliver to the US Air Force Phillips Laboratory.

This laser will be used in a long (600 ns) pulse mode for space object imaging.

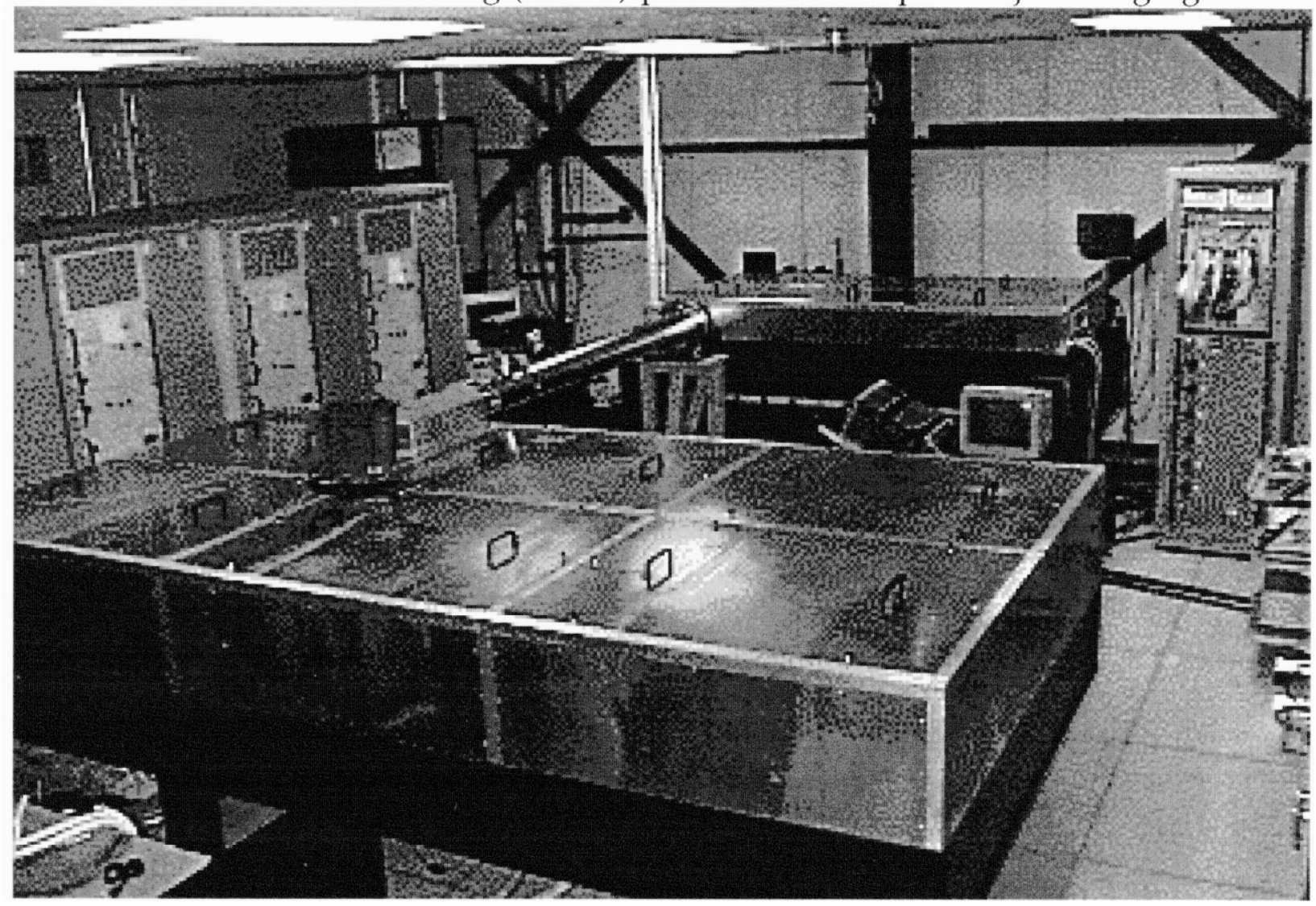

6

Figure $\%$. Completed laser, operational and ready for delivery to the US Air Force's Phillips Laboratory.

\section{High Throughput for Laser Peening}


The high average power available from the Nd:glass slab laser system enables for the first time a high throughput laser peening system. Assuming that $200 \mathrm{~J} / \mathrm{cm}^{2}$ is required to generate an effective $10 \mathrm{kBar}$ shock, the LLNL laser system, operating at $100 \mathrm{~J}$ per pulse and and $6 \mathrm{~Hz}$ repetition rate, will have a throughput capability in excess of $10,000 \mathrm{~cm}^{2}$ per hour for single pulse applications and $5,000 \mathrm{~cm}^{2}$ for dual pulse applications. Using the new APG-2 laser glass, the throughputs can be increased to $20,000 \mathrm{~cm}^{2}$ per hour and $10,000 \mathrm{~cm}^{2}$ per hour, respectively.

\section{Summary}

We have developed a class of laser system at the $100 \mathrm{~J}$ level with average power capability pushing toward $1 \mathrm{~kW}$. Its new technology includes uniformly pumped zig-zag slab gain media, master oscillator/power amplifier (MOPA) architectures and phase conjugation, to minimize the effects of thermal loading and correct the problems generated by it. This technology enables for the first time industrial application of a high throughput laser peening process.

This work was performed under the auspices of the U.S. Jepartment of Energy by Lawrence Livermore ilationat Laboratory under contract :io. W-7405Eng-48.

References:

1. B. P. Fairand and B. A. Wilcox, J. Appl Phys. 43 (1972) 3893

2. A. H. Clauer, B. P. Fairand and J. Holbrook, J. Appl Phys. 50 (1979) 1497

3. P. Peyre and R. Fabbro, Optical and Quantum Electronics 27 (1995) 1213-1229

4. S. R. Mannava, W. D. Cowie, A. E. McDaniel, "The Effects of Laser Shock Peening (LSP)_ on Airfoil FOD and High Cycle Fatigue", 1996 USAF Structural Integrity Program Conference, December 1996 


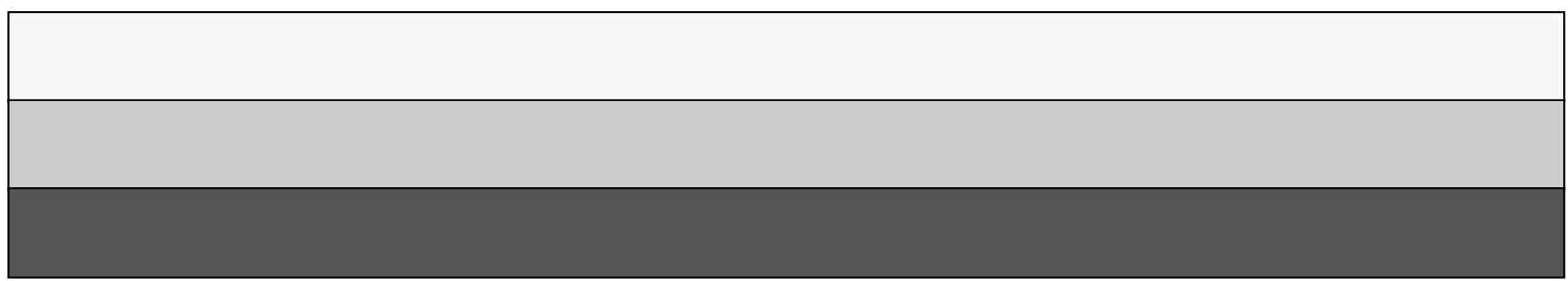

